\title{
3D skin bioprinting: future potential for skin regeneration
}

\author{
Anna Pasierb, Marta Jezierska, Aleksandra Karpuk, Joanna Czuwara, Lidia Rudnicka
}

Department of Dermatology, Medical University of Warsaw, Warsaw, Poland

Adv Dermatol Allergol 2022; XXXIX (5): 845-851

DOI: https://doi.org/10.5114/ada.2021.109692

\begin{abstract}
Skin provides protection against external agents and plays an essential role in maintaining the body homeostasis. Bioprinting as a novel strategy involves computer-controlled deposition of cells and scaffolds into a three-dimensional (3D) construction of skin. 3D bioprinting gives an opportunity to generate multi-layered vascularized skin grafts that can overcome the limitations of current skin substitutes. The main indication is treatment of troublesome wounds, especially severe burns and non-healing chronic lesions. Bioprinted skin equivalents offer a promising approach in the field of regenerative medicine. This review presents and discusses 3D skin construct formation, its limitations and modifications, and its usefulness.
\end{abstract}

Key words: 3D bioprinting, skin regeneration, tissue engineering.

\section{Introduction}

Skin as the outermost protective sheath of the human body is the ultimate multi-tasker "superorgan", performing many functions that are essential to overall well-being. It provides a barrier between the body and the external environment, inhibiting dermal penetration of external toxins and pathogens, mitigating external insults (e.g. UV radiation) and preventing desiccation. The dermis pulls together the extracellular matrix (ECM), containing mainly collagen produced by fibroblasts [1]. The arrangement of the ECM in the dermis contributes to skin's anisotropic nature, helping to assure the mechanical strength and elasticity of the tissue. Also, the dermis comprises numerous cell types supporting the connection between the skin and the rest of the body, including vasculature, sensory neurons, immune cells and various skin appendages, such as hair follicles, seborrheic and sweat glands [2].

Tissue engineering has been expanding as a novel strategy by employing the recent advances in multifarious fields such as polymer engineering, bioengineering, stem cell research and nanomedicine [3]. 3D printing has greatly evolved in the last 5 years and become one of the most promising techniques in tissue engineering. Bioprinting technology aims to generate accurately controlled organized assemblies and resemble the complex architectures of native tissues [3]. 3D bioprinting has formerly been used for the generation and transplanta- tion of several tissues, including multilayered skin, bone, vascular grafts, tracheal splints, heart tissue and cartilaginous textures [4-6].

In this review we introduce the 3D skin bioprinting method as a novel approach, its process step by step, recent achievements and future perspectives for clinical use in dermatology.

\section{Design and construction}

The technology of bioprinting involves computer-controlled deposition of cells and scaffolds into a 3D construction (Figure 1). Precise layer-by-layer placement of biological materials and living cells is possible due to the spatial control of the distribution of functional elements [7]. It is essential to choose the cellular component and compatible scaffolding to produce a proper functioning bioprint that will successfully mimic native tissue [8]. Biodegradable scaffolds play an important role in creating a 3D environment to induce tissue formation. The application of scaffolding materials together with stem cell technologies is believed to have enormous potential for tissue regeneration. The focus is on bone marrow stem cells. To provide mechanical strength, biological agents influencing stem cell fate could be added to the scaffold's compounds. Bone marrow stromal cells (mesenchymal stem cells) give rise to a variety of cell types: bone cells (osteocytes), cartilage cells (chondrocytes), fat

Address for correspondence: Joanna Czuwara MD, PhD, Department of Dermatology, Medical University of Warsaw, 82A Koszykowa St, 02-008 Warsaw, Poland, e-mail: joanna.czuwara@wum.edu.pl Received: 21.04.2021, accepted: 21.07.2021. 


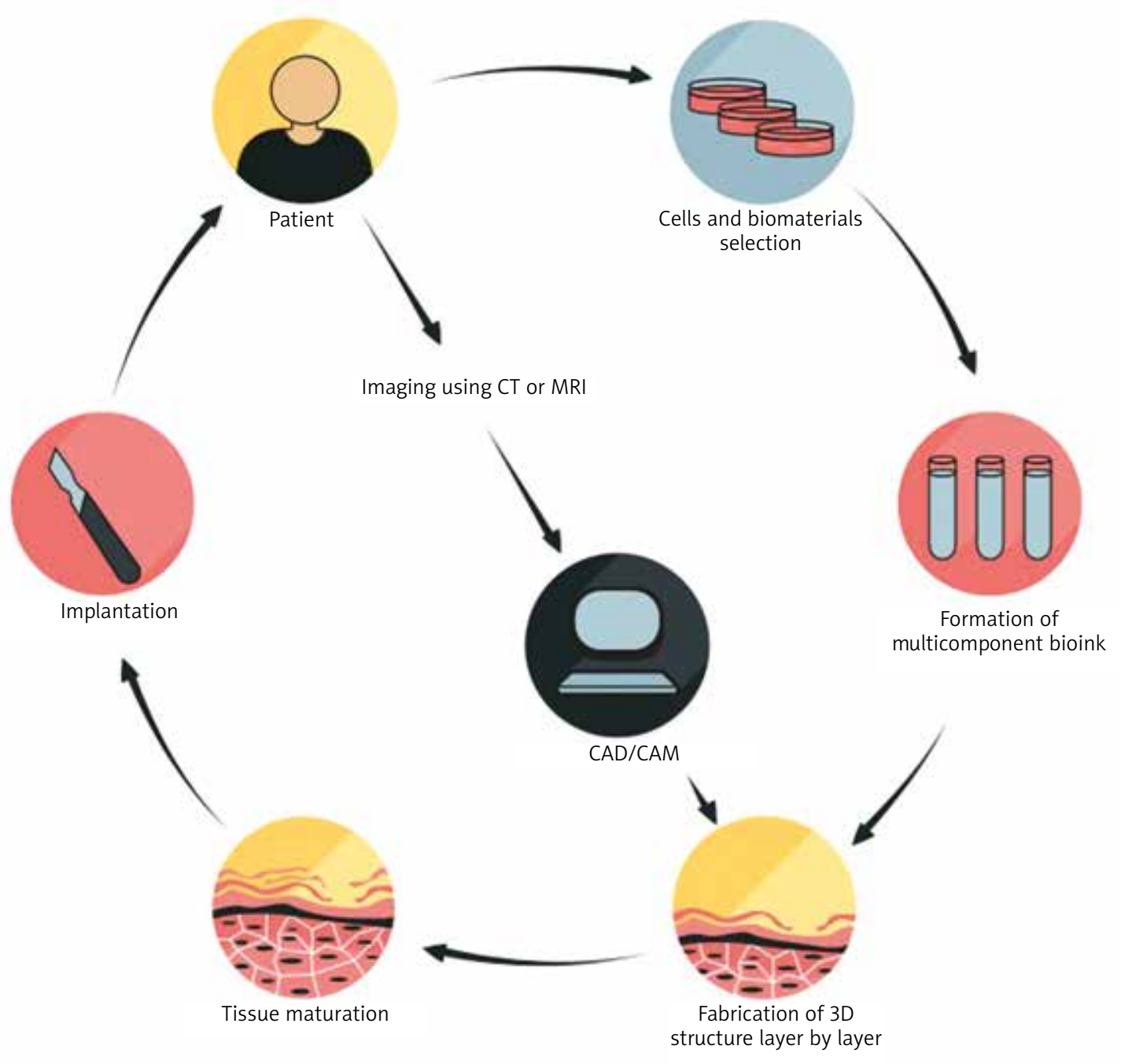

$\mathrm{CT}$ - computed tomography, MRI - magnetic resonance imaging, CAD/CAM - computer-aided design/computer-aided manufacturing.

Figure 1. Three-dimensional tissue manufacturing procedure. The diagram illustrates successive phases of artificial tissue design and implantation. The use of patient-derived cells and properly matched biomaterials creates the opportunities for personalization of the transplant

cells (adipocytes), and other kinds of connective tissue cells such as those in tendons. The fundamental roles of adult stem cells in a living organism are to preserve and repair the tissue where they are found and to form specialized cell types of other tissues, which is known as transdifferentiation or plasticity $[4,5]$.

Scaffolding materials, such as hydrogels, and synthetic or natural polymers, in association with additives (growth factors and chemical molecules), together determine bioink. The origins of the cells employed in bioprinting technology are primary cells, cell lines and stem cell-derived cells. The primary cells, although able to recapitulate the function of certain tissue, have restricted expansion capacity and limited lifespan. Hence, the usage of stem cells in skin equivalents has the potential to grow into specific somatic cell lineages under the influence of patterning signals [9]. The most valuable cell reservoir in skin bioprinting is the autologous cells derived from the patient [3] (Figure 1). Proper selection of bioinks is of key importance in fabricating functional tissue structures. The scaffold of the construct is a cell-laden biomaterial which acts as the ink for bioprinters and provides a biocompatible gel that has to maintain suitable properties to match the tissue [7]. Constructs should have certain physical, chemical and biological features such as adequate mechanical stability and structural rigidity, appropriate pore size, interconnected channels and pores for the cell migration and fluid transport $[3,10]$. Bioink is critical to ensure the viability and responsiveness of cells in 3D-printed architectures and can be simply di- 
vided into two types, which are natural biopolymer and synthetic one. Many scientists focus on natural polymers, in order to more accurately mimic the native extracellular matrix, leading to more favourable cell responses. Some of the natural polymers that are currently explored for bioprinting include collagen, gelatin, alginate, fibrin, hyaluronan and dextran. However, synthetic polymers ensure mechanical firmness, tailored porosity cross-linking abilities and amplified cell signalling capabilities, but the main limitation is lack of biological activity. Examples of artificial polymers are polyethylene glycol, polyglycolic acid and polylactic acid [11-13].

\section{Steps in the process}

The process of three-dimensional tissue production begins with accurate imaging of the skin lesion (Figure 2), for which computed tomography (CT) or magnetic resonance imaging (MRI) is commonly used. Although CT gives the opportunity to obtain images with relatively good quality in a short time, it is associated with a high dose of harmful ionizing radiation. Computer-aided design (CAD) enables the creation of a virtual model of the requested tissue and also possibility to precisely analyse and assess the construct's behaviour in diverse conditions. The CAD/CAM software offers the opportunity to convert digital ideas into the code comprehendible for bioprinters [14]. The next step is using a bioprinter to deposit layer by layer biomaterials to form the spatial imitation of the skin (Figure 2).
There are several printing strategies, which include inkjet-based bioprinting, microextrusion bioprinting, laser-assisted bioprinting (LAB), stereolithography (SLA) and microfluidic bioprinting. The inkjet-based bioprinter operates on principles analogous to conventional 2D printers in which the classic ink is replaced by the biological materials, whereas a specially prepared substrate performs the role of the sheet of paper. Droplets of cells placed in organic dissolvent are applied under precise computer control [15]. Considering the driving forces needed for cell deposition, there are three methods of pressure generation in drop-on-demand technique, i.e. thermal, piezoelectric and electrostatic $[16,17]$. The advantages of inkjet-based bioprinting are cost-effective, relatively fine resolution and high velocity of cell dissemination. The viscosity of the bioink remains a problem. It forces the usage of high frequencies, which may affect cell viability [7]. Extrusion-based bioprinting can produce large structures in both horizontal and vertical orientations. It has the capability of printing highly viscous bioinks that contain high cell densities [18]. The main obstacle is low resolution and the shear stress that may affect cell viability [19]. Lee et al. fabricated a multi-layered skin-like tissue structure with the inclusion of fibroblasts and keratinocytes using both inkjet and extrusion printing processes [20]. Another method is based on the use of a pulsed laser beam and the absorbing ability of the ribbon structure after exposure to a focused pulsed laser [21]. Its biggest asset is the facility to transfer cells onto subsoil at high density, securing their survival [22]. The resolution of this technique can vary with changing pa-

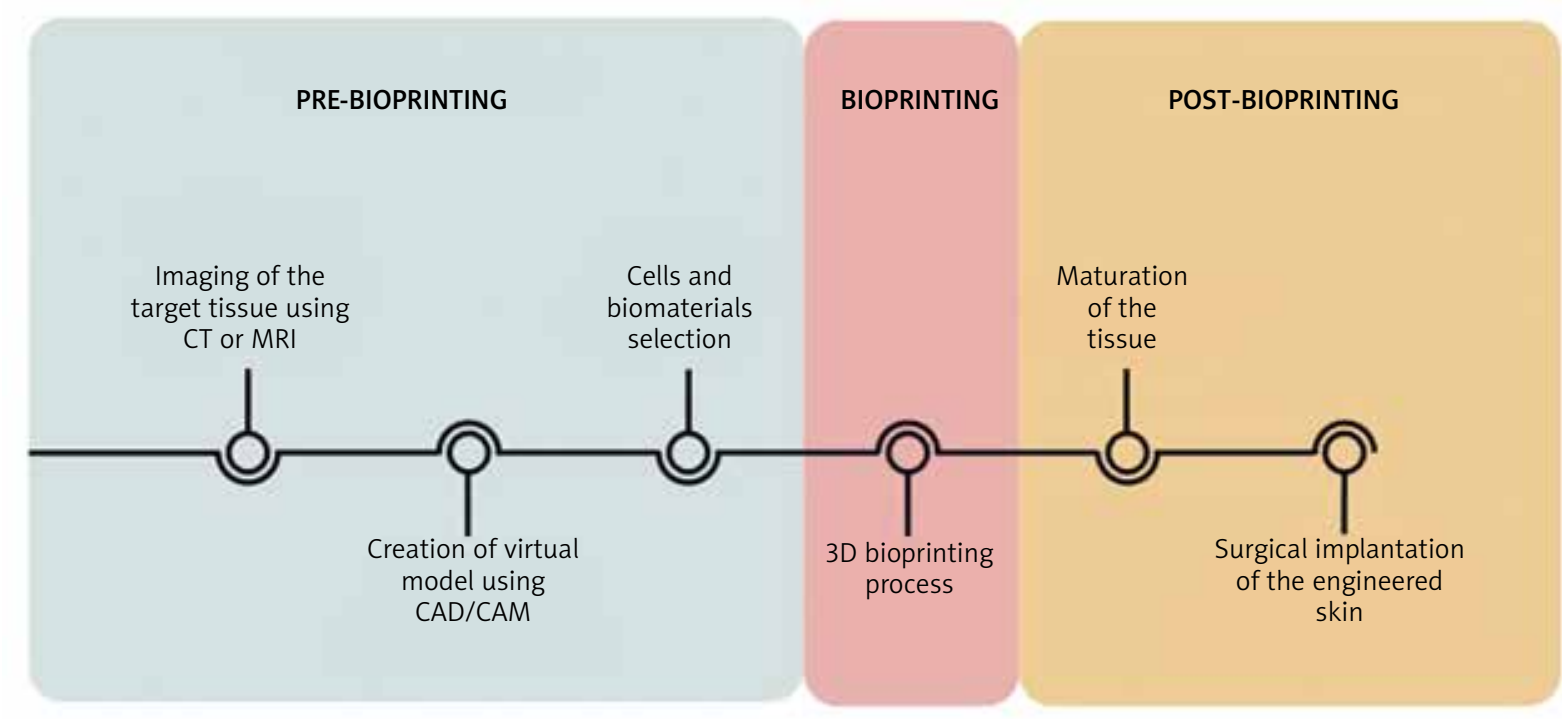

CT - computed tomography, MRI - magnetic resonance imaging, CAD/CAM - computer-aided design/computer-aided manufacturing.

Figure 2. Steps in the process of 3D bioprinting. The process of 3D skin fabrication consists of three stages: pre-bioprinting, bioprinting and post-bioprinting. This process is extremely precise and each stage is strictly defined in time. The technique involves the use of the most accurate imaging methods, modern IT programs and tissue engineering approaches 
rameters, such as viscosity, printing speed, pattern topology and laser pulse energy. Stereolithography is a technique in which the precursor hydrogel is crosslinked via photoirradiation. The process of hardening the hydrogel is called photocuring, which is aided by the addition of photoinitiators. SLA printing has high accuracy and precision fabrication that can print light-sensitive bioinks. The main disadvantage of SLA is the limited number of biocompatible materials and time-consuming UV crosslinking process, which can be deleterious to biological components [23]. Microfluidic-based 3D bioprinting systems can be used to control cell and molecule deposition, flow, mixing and gradient in building 3D structures. One of the challenges is the difficulty to maintain precision, especially at the corners of resulting constructs because of the difficulty in maintaining smooth non-fluctuating flow and pressure in the device $[23,24]$. All of these printing strategies can be used alone or in combination to achieve the most satisfactory effect [14, 25, 26].

\section{Current views on skin substitutes}

Currently, harmed skin can be replaced with the patient's own skin or tissue donated by another human or another species. These methods, widely used in clinical practice, are known as autografts, allografts and xenografts, respectively. The transplantation of allogeneic skin grafts is associated with a risk of destruction as a consequence of an inflammatory response [27]. Certainly the best option of skin replacement is the use of autografts, though it requires sufficient volumes of skin harvest sites and is associated with a secondary wound creation [28]. The increasing demand resulted in rapid development of tissue-engineered skin substitutes. Epicel, Epidex and Myskin are some examples of epidermal substitutes. Epicel and Epidex are organised by autologous keratinocytes obtained from the outer root sheath of scalp hair follicles. Myskin consists of a surface coated silicon substrate, covered with sub-confluent autologous keratinocytes which improves handling application and decreases the cell culture time [29].

Engineered dermal substitutes provide appropriate configuration and surface area for effective epidermal engraftment. Some currently available dermal substitutes are AlloDerm, Dermagraft Integra and Matriderm. AlloDerm is an acellular human dermis produced by the removal of the epidermis and extraction of fibroblasts from the dermis while the collagen bundles or the basement membrane remain unchanged. On the other hand, Dermagraft as an engineered dermal substitute contains cryopreserved human fibroblast cells from newborn foreskin tissue. Cost and antigenic response are the main disadvantages of this graft. Integra consists of a porous matrix of cross-linked bovine type I tendon collagen, shark chondroitin-6-sulfate glycosaminoglycan and a semi-permeable polysiloxane and provides promising advantages including long shelf life, simple handling, comfortability for various anatomical sites, low risks of immunogenic response and disease transposition and reduced rates of constriction and scarring. Matriderm was designed as a 3D matrix consisting of a collagen matrix coated with an elastin hydrolysate from the ligament, similar to the structure of the human dermis. It acquires more elastic properties similar to natural skin [13, 29].

Dermo-epidermal substitutes are composed of two layers comprising keratinocytes on fibroblast-containing dermal substitutes. Apligraf, the first approved composite substitute to heal both diabetic foot ulcers and venous leg ulcers, consists of two layers and comprises in the lower dermal layer bovine type I collagen and allogeneic neonatal fibroblasts, which produce additional matrix proteins. The upper epidermal layer is made of allogeneic neonatal keratinocytes. OrCel is, like Apligraf, a bilayered cellular matrix, in which human neonatal foreskin allogeneic epidermal keratinocytes and dermal fibroblasts are cultured in two individual layers into a type I bovine collagen sponge. It is composed of a biocompatible matrix which is shown contain cytokines and growth factors - all suitable for host cell migration and wound healing [30-32].

\section{Achieving functionality}

The presence of appendages and blood vessels is essential for proper implantation and supplementation of the tissue with oxygen, nutrients and growth factors.

\section{Sweat glands}

When damaged, it is almost impossible to rebuild and restore the function of sweat glands. In the study by Huang et al. using 3D bioprinting technology, creation of sweat glands could be achieved due to specific cell differentiation. Furthermore, it was proven that the functional restoration of sweat glands is possible by applying bioprinted tissue in burned paws of mice (Table 1) $[33,34]$.

\section{Hair follicles}

Proper morphogenesis of the hair depends on the activation of the hair follicle stem cells (HFSCS). Depending on the ever-changing external environment, HFSCs can be directly or indirectly stimulated to remain quiescent or to enter the next regeneration cycle [35]. Previous studies demonstrated human bioengineered hair follicle regeneration after intracutaneous application of adult follicle-derived stem cells into the skin of nude mice. After 21 days, the researchers succeeded in achieving growth of pigmented hair shaft $[36,37]$. Abaci et al. designed a human skin construct (HSC) with the spatial distribution of human hair papilla cells that had the conformation of natural hair follicles. Their innovative method make it possible to create 
Table 1. Recent milestones in 3D bioprinting

\begin{tabular}{|c|c|c|}
\hline Research achievement & Research group & Year \\
\hline Bioprinted skin with sweat glands & Huang et al. & 2015 \\
\hline Perfusion of thick bioprinted tissue with embedded vasculature & Kolesky et al. & 2016 \\
\hline Reconstruction of hair follicle using human hair papilla cells in human skin construct & Abaci et al. & 2018 \\
\hline Fabrication of pigmented construct using fibroblasts, keratinocytes and melanocytes & Min et al. & 2018 \\
\hline $\begin{array}{l}\text { Generation of 3D construct using Muse cells differentiating into melanocytes, fibroblasts and } \\
\text { keratinocytes }\end{array}$ & Yamauchi eet al. & 2018 \\
\hline Organization of human endothelial cells and pericytes into microvascular networks prior to engraftment & Baltazar et al. & 2019 \\
\hline 3D construct with sebaceous glands producing sebum with the right composition & de Bengy et al. & 2019 \\
\hline
\end{tabular}

a bioprint comprising hair entirely from ex vivo cultured human cells and revealed that previous vascularization of the HSC using human umbilical endothelial cells and fibroblasts resulted in increased survival of the graft and efficient hair growth in mice (Table 1) [38].

\section{Sebaceous glands}

Previous research revealed that specially prepared human sebocyte cell lines in multilayer culture spontaneously differentiated into a sebaceous-like structure. These cells presented sebaceous markers on their surface and were able to produce lipid vesicles [39]. The demonstrated ex vivo 3D construct showed the ability to produce sebum with the right composition (Table 1).

\section{Vascularization and vessel formation}

In order for the 3D bioprint to mimic physiological skin and preserve its functionality, integration with the host's tissues and sufficient vascularization with accurate cell adhesion and accessibility of oxygen and nutrients, integration with the host's tissues is essential $[40,41]$. The process of angiogenesis depends on the synergistic cooperation between selected cells interacting with each other via growth factors and cytokines [42]. An alternative approach is using a bioactive bioink, which could be capable of self-assembly into capillarylike networks. Therefore there is a great need to create a bioprint with pre-formed vessels or components suitable for their formation after implantation [43]. Achieving tissue perfusion is a major feature providing durability of constructs thicker than $1 \mathrm{~mm}$. Kolesky et al. made an attempt to meet these requirements by coprinting multiple inks containing mainly human mesenchymal stem cells (hMSCs) and human neonatal dermal fibroblasts (hNDFs) (Table 1) [44].

Baltazar et al. discovered that human endothelial cells along with pericytes organized themselves into microvascular networks prior to engraftment. The effectiveness of this approach was confirmed by connecting microvessels of the host with vasculature in the bioprinted tissue. Perfusion of the 3D-engineered graft was achieved in 4 weeks (Table 1) [45].

\section{Pigmentation and melanin production}

The individual complexion phenotype for every human being is contingent on the ability of melanocytes to produce melanin. The melanin collects around the nuclei and prevents the harmful impact of solar UV radiation [46]. The incorporation of melanocytes into the fabricated skin became a reality by means of 3D bioprinting technology. The study by Min et al. enables the creation of bioprint pigmented constructs using fibroblasts, keratinocytes and melanocytes with a proper architecture through correct attachment and maturation of the components, allowing new approaches that will be less time-consuming and more practically useful [47].

Bearing in mind the immense prevalence of pluripotent stem cells in tissue engineering, attention has begun to be paid to the possibility of their transformation into melanocytes. A promising idea is multilineage-differentiating stressenduring (Muse) cells with capacity to differentiate into all of the three germ layers, i.e. ectoderm, mesoderm and endoderm (Table 1) [48]. The biggest advantage of Muse cells is easy accessibility, because they originally residue in the dermis, adipose tissue and bone marrow. It was reported that Muse cell-derived melanocytes expressed specific surface markers after being transplanted as 3D bioprinted tissue to immunodeficient mice [49]. Yamauchi et al. established the technique of gaining not only melanocytes, but also keratinocytes and fibroblasts from Muse cells in vitro and succeeded in generating 3D constructs using these elements, which maintained their functionality [50].

\section{Potential practical application for clinicians and future perspectives}

The technology of bioprinting has the potential to revolutionize contemporary regenerative medicine. There are vast numbers of patients, victims of severe burns or patients suffering from chronic ulcers, for whom this method could bring considerable benefits. Simple excision of disfiguring marks is associated with creating a secondary wound [51]. For all those circumstances the 3D-bioprinting method is a promising approach, as it has been proven to facilitate faster wound closure. By taking advantage 
of tissue regeneration techniques it becomes possible to imitate characteristic inflammatory profiles [52, 53], to study drug-related toxicity [54], and also to investigate the pathological mechanism of some skin diseases, including psoriasis and atopic dermatitis [55-57]. Cosmetic and pharmaceutical industries testing on laboratory animals raises many ethical considerations. Since European Union regulations imposed a prohibition to test cosmetic products and ingredients on animals, consideration of tissue engineering methods in meeting these requirements may result in increased spatial and chemical conformity with human skin and also reproducibility [58]. The incorporation of additional cell types including melanocytes into 3D equivalents makes it possible to study the impact of UV radiation and susceptibility to photodamage [59]. Potential insertion of genetically altered or cancer cells into a printed tissue could extend the knowledge about neoplasm proliferation and migration, but also about metastatic abilities of the tumour and drug response [60, 61].

One of the long-term goals of 3D bioprinting is the ability to produce clinical-grade tissues. However, translation of these 3D printed implants and tissues to clinical applications will require long-term in vivo testing to investigate the functionality and integration of constructs [13]. Compared to machining prototypes, 3D printing is inexpensive and quicker at creating parts, as the part can be finished in hours, allowing for each design modification to be completed at a much more efficient rate. The manufacturing process can also reduce costs of materials as it only uses the amount of material required for the part itself, with little or no wastage. Significant inconveniences of 3D printing are consumption of large amounts of energy and difficulty in maintaining the cell environment, resulting in the death of many cells $[13,62]$.

\section{Conclusions}

Over the past two decades, significant progress has been made in the field of skin engineering. A big challenge for tissue engineering is producing three-dimensional, vascularized cellular constructs of clinically relevant size, shape and structural integrity. Bioprinting facilitates concurrent engineering design that spans the micro- and macro scales, thus enabling the fabrication of constructs that can better satisfy the various requirements of a natural niche for skin cells. However, the use of bioprinting for skin reconstruction is very propitious: it will allow accurate placement of cell types and exact and reproducible fabrication of constructs to replace the injured skin. Tomorrow's method is bioprinting, which will take tissue engineers and clinicians far beyond current expectations for regenerative medicine and dermatology.

\section{Conflict of interest}

The authors declare no conflict of interest.

\section{References}

1. Supp DM, Boyce ST. Engineered skin substitutes: practices and potentials. Clin Dermatol 2005; 23: 403-12.

2. Yan WC, Davoodi P, Vijayavenkataraman S, et al. 3D bioprinting of skin tissue: from pre-processing to final product evaluation. Adv Drug Deliv Rev 2018; 132: 270-95.

3. Augustine R. Skin bioprinting: a novel approach for creating artificial skin from synthetic and natural building blocks. Prog Biomater 2018; 7: 77-92.

4. Kaur A, Midha S, Giri S, Mohanty S. Functional skin grafts: where biomaterials meet stem cells. Stem Cells Int 2019; 2019: 1-20.

5. Tarassoli SP, Jessop ZM, Al-Sabah A, et al. Skin tissue engineering using 3D bioprinting: an evolving research field. J Plast Reconstr Aesthet Surg 2018; 71: 615-23.

6. Miguel SP, Cabral CSD, Moreira AF, Correia IJ. Production and characterization of a novel asymmetric 3D printed construct aimed for skin tissue regeneration. Colloids Surf B Biointerfaces 2019; 181: 994-1003.

7. Murphy SV, Atala A. 3D bioprinting of tissues and organs. Nat Biotechnol 2014; 32: 773-85.

8. Pescosolido L, Schuurman W, Malda J, et al. Hyaluronic acid and dextran-based semi-IPN Hydrogels as biomaterials for bioprinting. Biomacromolecules 2011; 12: 1831-8.

9. Takagi R, Ishimaru J, Sugawara A, et al. Bioengineering a 3D integumentary organ system from iPS cells using an in vivo transplantation model. Sci Adv 2016; 2: e1500887.

10. Koch L, Deiwick A, Franke A, et al. Laser bioprinting of human induced pluripotent stem cells - the effect of printing and biomaterials on cell survival, pluripotency, and differentiation. Biofabrication 2018; 10: 035005.

11. Ahadian S, Khademhosseini A. A perspective on 3D bioprinting in tissue regeneration. Bio-Des Manuf 2018; 1: 157-60.

12. Wang R, Wang Y, Yao B, et al. Beyond 2D: 3D bioprinting for skin regeneration. Int Wound J 2019; 16: 134-8.

13. Ishack S, Lipner SR. A review of 3-dimensional skin bioprinting techniques: applications, approaches, and trends. Dermatol Surg 2020; 46: 1500-5.

14. Crook JM ed. 3D Bioprinting: Principles and Protocols. Springer US, New York, NY 2020.

15. Matai I, Kaur G, Seyedsalehi A, et al. Progress in 3D bioprinting technology for tissue/organ regenerative engineering. Biomaterials 2020; 226: 119536.

16. Cui X, Boland T, D'Lima DD, Lotz MK. Thermal inkjet printing in tissue engineering and regenerative medicine. Recent Pat Drug Deliv Formul 2012; 6: 149-55.

17. Li X, Chen J, Liu B, et al. Inkjet printing for biofabrication. In: 3D Printing and Biofabrication. Ovsianikov A, Yoo J, Mironov V (eds). Springer International Publishing, Cham 2018; 283-301.

18. Bishop ES, Mostafa S, Pakvasa M, et al. 3-D bioprinting technologies in tissue engineering and regenerative medicine: current and future trends. Genes Dis 2017; 4: 185-95.

19. Ozbolat IT, Hospodiuk M. Current advances and future perspectives in extrusion-based bioprinting. Biomaterials 2016; 76: 321-43.

20. Lee W, Debasitis JC, Lee VK, et al. Multi-layered culture of human skin fibroblasts and keratinocytes through three-dimensional freeform fabrication. Biomaterials 2009; 30: 1587-95.

21. Gu Z, Fu J, Lin H, He Y. Development of 3D bioprinting: from printing methods to biomedical applications. Asian J Pharm Sci 2020; 15: 529-57.

22. Varkey M, Visscher DO, van Zuijlen PPM, et al. Skin bioprinting: the future of burn wound reconstruction? Burns Trauma 2019; 7: 4 . 
23. Davoodi E, Sarikhani E, Moutazerian H, et al. Extrusion and microfluidic-based bioprinting to fabricate biomimetic tissues and organs. Adv Mat Tech 2020; 5: 1901044.

24. Hasan A, Paul A, Vrana NE, et al. Microfluidic techniques for development of 3D vascularized tissue. Biomaterials 2014; 35: 7308-25.

25. Ozbolat I, Yu Y. Bioprinting towards organ fabrication: challenges and future trends. IEEE Trans Biomed Eng 2013; 60: 691-9.

26. Chen Y, Zhang J, Liu X, et al. Noninvasive in vivo 3D bioprinting. Sci Adv 2020; 6: eaba7406.

27. Benichou G, Yamada Y, Yun SH, et al. Immune recognition and rejection of allogeneic skin grafts. Immunotherapy 2011; 3: 75770.

28. Zöller N, Valesky E, Butting M, et al. Clinical application of a tissue-cultured skin autograft: an alternative for the treatment of non-healing or slowly healing wounds? Dermatology 2014; 229: 190-8.

29. Savoji H, Godau B, Hassani MS, et al. Skin tissue substitutes and biomaterial risk assessment and testing. Front Bioeng Biotechnol 2018; 6: 86.

30. Chocarro-Wrona C, López-Ruiz E, Perán M, et al. Therapeutic strategies for skin regeneration based on biomedical substitutes. J Eur Acad Dermatol Venereol 2019; 33: 484-96.

31. Jorgensen AM, Varkey M, Gorkun A, et al. Bioprinted skin recapitulates normal collagen remodeling in full-thickness wounds. Tissue Eng Part A 2020; 26: 512-26.

32. Albanna M, Binder KW, Murphy SV, et al. In situ bioprinting of autologous skin cells accelerates wound healing of extensive excisional full-thickness wounds. Sci Rep 2019; 9: 1856.

33. Huang S, Yao B, Xie J, Fu X. 3D bioprinted extracellular matrix mimics facilitate directed differentiation of epithelial progenitors for sweat gland regeneration. Acta Biomater 2016; 32: 170-7.

34. Pedde RD, Mirani B, Navaei A, et al. Emerging biofabrication strategies for engineering complex tissue constructs. Adv Mater 2017; 29: 1606061.

35. Chen CL, Huang WY, Wang EHC, et al. Functional complexity of hair follicle stem cell niche and therapeutic targeting of niche dysfunction for hair regeneration. J Biomed Sci 2020; 27: 43.

36. Toyoshima K, Asakawa K, Ishibashi N, et al. Fully functional hair follicle regeneration through the rearrangement of stem cells and their niches. Nat Commun 2012; 3: 784.

37. Asakawa K, Toyoshima K, Ishibashi N, et al. Hair organ regeneration via the bioengineered hair follicular unit transplantation. Sci Rep 2012; 2: 424.

38. Abaci HE, Coffman A, Doucet $Y$, et al. Tissue engineering of human hair follicles using a biomimetic developmental approach. Nat Commun 2018; 9: 5301.

39. Barrault C, Dichamp I, Garnier J, et al. Immortalized sebocytes can spontaneously differentiate into a sebaceous-like phenotype when cultured as a 3D epithelium: letter to the Editor. Exp Dermatol 2012; 21: 314-6.

40. Richards D, Jia J, Yost M, et al. 3D bioprinting for vascularized tissue fabrication. Ann Biomed Eng 2017; 45: 132-47.

41. Hendrickx B, Vranckx JJ, Luttun A. Cell-based vascularization strategies for skin tissue engineering. Tissue Eng Part B Rev 2011; 17: 13-24.

42. Xiong S, Zhang X, Lu P, et al. A gelatin-sulfonated silk composite scaffold based on 3d printing technology enhances skin regeneration by stimulating epidermal growth and dermal neovascularization. Sci Rep 2017; 7: 4288.

43. Patra S, Young V. A review of $3 D$ printing techniques and the future in biofabrication of bioprinted tissue. Cell Biochem Biophys 2016; 74: 93-8.
44. Kolesky DB, Homan KA, Skylar-Scott MA, Lewis JA. Three-dimensional bioprinting of thick vascularized tissues. Proc Natl Acad Sci 2016; 113: 3179-84.

45. Baltazar T, Merola J, Catarino C, et al. Three dimensional bioprinting of a vascularized and perfusable skin graft using human keratinocytes, fibroblasts, pericytes, and endothelial cells. Tissue Eng Part A 2020; 26: 227-38.

46. LaBerge GS, Duvall E, Grasmick Z, et al. Recent advances in studies of skin color and skin cancer. Yale J Biol Med 2020; 93: 69-80.

47. Min D, Lee $W$, Bae IH, et al. Bioprinting of biomimetic skin containing melanocytes. Exp Dermatol 2018; 27: 453-9.

48. Yamauchi T, Yamasaki K, Tsuchiyama K, et al. A quantitative analysis of multilineage-differentiating stress-enduring (Muse) cells in human adipose tissue and efficacy of melanocytes induction. J Dermatol Sci 2017; 86: 198-205.

49. Tsuchiyama K, Wakao S, Kuroda Y, et al. Functional melanocytes are readily reprogrammable from multilineage-differentiating stress-enduring (Muse) cells, distinct stem cells in human fibroblasts. J Invest Dermatol 2013; 133: 2425-35.

50. Yamauchi T, Yamasaki K, Tsuchiyama K, Aiba S. Artificial pigmented human skin created by muse cells. In: Muse Cells. Dezawa M (ed.). Springer Japan, Tokyo 2018; 255-71.

51. El-Serafi AT, El-Serafi IT, Elmasry M, et al. Skin regeneration in three dimensions, current status, challenges and opportunities. Differentiation 2017; 96: 26-9.

52. Yang Q, Lian Q, Xu F. Perspective: fabrication of integrated organ-on-a-chip via bioprinting. Biomicrofluidics 2017; 11: 031301.

53. Wufuer M, Lee G, Hur W, et al. Skin-on-a-chip model simulating inflammation, edema and drug-based treatment. Sci Rep 2016; 6: 37471.

54. Wei Z, Liu X, Ooka M, et al. Two-dimensional cellular and threedimensional bio-printed skin models to screen topical-use compounds for irritation potential. Front Bioeng Biotechnol 2020; 8: 109.

55. Lorthois I, Simard M, Morin S, Pouliot R. Infiltration of T cells into a three-dimensional psoriatic skin model mimics pathological key features. Int J Mol Sci 2019; 20: 1670.

56. Clarysse K, Pfaff CM, Marquardt Y, et al. JAK1/3 inhibition preserves epidermal morphology in full-thickness 3D skin models of atopic dermatitis and psoriasis. J Eur Acad Dermatol Venereol 2019; 33: 367-75.

57. Liu X, Michael S, Bharti K, et al. A biofabricated vascularized skin model of atopic dermatitis for preclinical studies. Biofabrication 2020; 12: 035002.

58. Derr K, Zou J, Luo K, et al. Fully three-dimensional bioprinted skin equivalent constructs with validated morphology and barrier function. Tissue Eng Part C Methods 2019; 25: 334-43.

59. Randall MJ, Jüngel A, Rimann M, Wuertz-Kozak K. Advances in the biofabrication of 3D sin in vitro: healthy and pathological models. Front Bioeng Biotechnol 2018; 6: 154.

60. Vultur A, Schanstra T, Herlyn M. The promise of 3D skin and melanoma cell bioprinting. Melanoma Res 2016; 26: 205-6.

61. Vijayavenkataraman S, Lu WF, Fuh JYH. 3D bioprinting of skin: a state-of-the-art review on modelling, materials, and processes. Biofabrication 2016; 8: 032001.

62. Ng W, Wang S, Yeong W, et al. Skin bioprinting: impending reality or fantasy? Trends Biotech 2016; 34: 689-99. 